\title{
Thoracoscopic double aortic arch division with Harmonic scalpel: A case report
}

\author{
Pablo Laje, MD, and Marcelo Martínez-Ferro, MD, Buenos Aires, Argentina
}

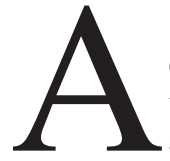

double aortic arch incidentally found during the course of a thoracoscopic repair of a type III esophageal atresia was successfully repaired by dividing the nondominant arch with the Harmonic scalpel device (Ultrashears, Autosonix Ultrasonic System; Tyco Healthcare, Mansfield, Mass) on day 1 after birth. The association of double aortic arch and esophageal atresia has been reported many times in the literature ${ }^{1}$ and has important implications, because a proper esophageal reconstruction can be achieved only after the vascular anomaly has been corrected.

\section{Clinical Summary}

A boy weighing $2.080 \mathrm{~kg}$ was born at 36 weeks of gestational age. No prenatal images were done. Immediately after birth, all attempts to place a nasogastric tube were unsuccessful, and a plain x-ray film showed air throughout the bowel loops. The patient was transferred to our institution in stable condition for further treatment. An echocardiogram done soon after arrival confirmed a left orientation of the aortic arch, but was otherwise unremarkable. On day 1 after birth, a thoracoscopic repair of the type III esophageal atresia was performed. We initially identified and ligated the tracheoesophageal fistula. When we attempted to dissect the upper esophageal pouch, we found an aberrant arterial vessel connecting the right aspect of the aortic arch with the descending aorta, in direct contact with the posterior wall of the trachea, resembling the exact configuration of the posterior arch of a double aortic arch (Figure 1). It was located right where the esophageal anastomosis was going to be done. We temporarily clamped the aberrant vessel and checked blood pressure, temperature, arterial pulse, pulse oximetry, and color changes in all limbs. There were no variations whatsoever in any of these parameters, meaning that this posterior arch was definitely nondominant and therefore had to be treated. We then divided it with the 5-mm Harmonic scalpel (Figure 2), leaving two well-sealed stumps and relieving the posterior wall of the trachea from the vascular compression. After this, the upper esophageal pouch was dissected, and the end-to-end esophageal anastomosis was performed without difficulties. A $12 \mathrm{~F}$ chest tube was left in place. Total operative time was 130 minutes. The patient was extubated 36 hours after the operation. No intraoperative or postoperative complications were observed.

From the Division of General Pediatric Surgery, National Pediatric Hospital "JP Garrahan," Buenos Aires, Argentina.

Received for publication Oct 25, 2006; accepted for publication Oct 30, 2006.

Address for reprints: Pablo Laje, MD, 2979 W School House Lane, Apt K-1103, Philadelphia, PA 19144 (E-mail: laje@email.chop.edu).

J Thorac Cardiovasc Surg 2007;133:819-20

$0022-5223 / \$ 32.00$

Copyright () 2007 by The American Association for Thoracic Surgery

doi:10.1016/j.jtcvs.2006.10.056

\section{Discussion}

The Harmonic scalpel was designed many years ago with the aim of providing good hemostasis and little tissue damage during dissection owing to its ability to generate localized heat. In the cardiac surgery field, it is currently used for radial, thoracic, and gastroepiploic artery harvesting, pericardial resections, and tissue dissection during redo cases. $^{2-4}$ In other surgical specialties its applications are widely diverse, from division of splenic vessels, to thoracic duct division, thoracoscopic sympathectomies, lung resections, hepatectomies, pancreatic resections, partial and total nephrectomies, varicocelectomies, and hysterectomies, among many others. It has been proved to be a safe tool in terms of hemostasis, even when used to divide large systemic arteries, such as the splenic artery, or the feeding arteries of pulmonary sequestrations. ${ }^{5}$ There is no specific information regarding the vessel size above which the Harmonic scalpel should not be used, but the general rule for this and other vessel-sealing devices is that the vessel being treated should thoroughly fit in between the jaws of the instrument after being clamped, with no segments remaining outside the grasping area. In our case, based on the size of the instruments we were using, we estimate that the aberrant vessel was probably 2 to 2.5 $\mathrm{mm}$ in diameter, occupying about a third of the grasping area.

There is still some controversy as regards the thoracoscopic approach for major vascular surgery and cardiac surgery, mainly because, in the event of major bleeding, the loss of visualization may prevent an appropriate control of the hemorrhage while converting to open surgery. As far as we know, a randomized comparative study on the minimally invasive surgical treatment of vascular rings has not yet been reported, so conclusions are still

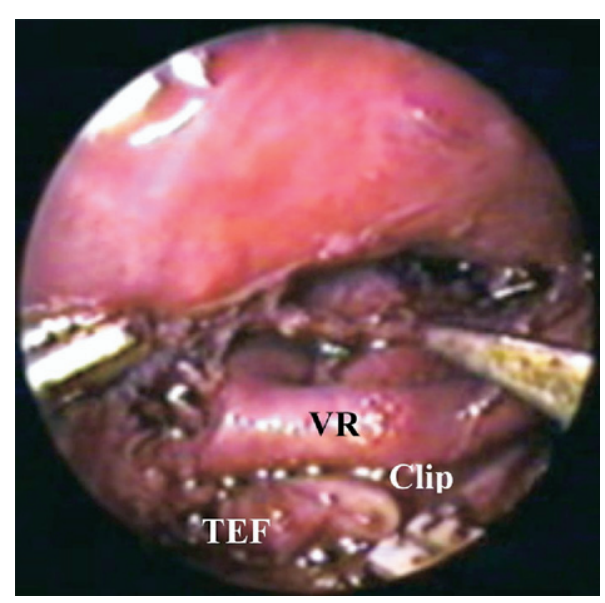

Figure 1. The abnormal vessel (VR) is adjacent to the posterior wall of the trachea, proximal to the tracheoesophageal fistula (TEF), connecting the descending aorta with the right aspect of the anterior aortic arch. 


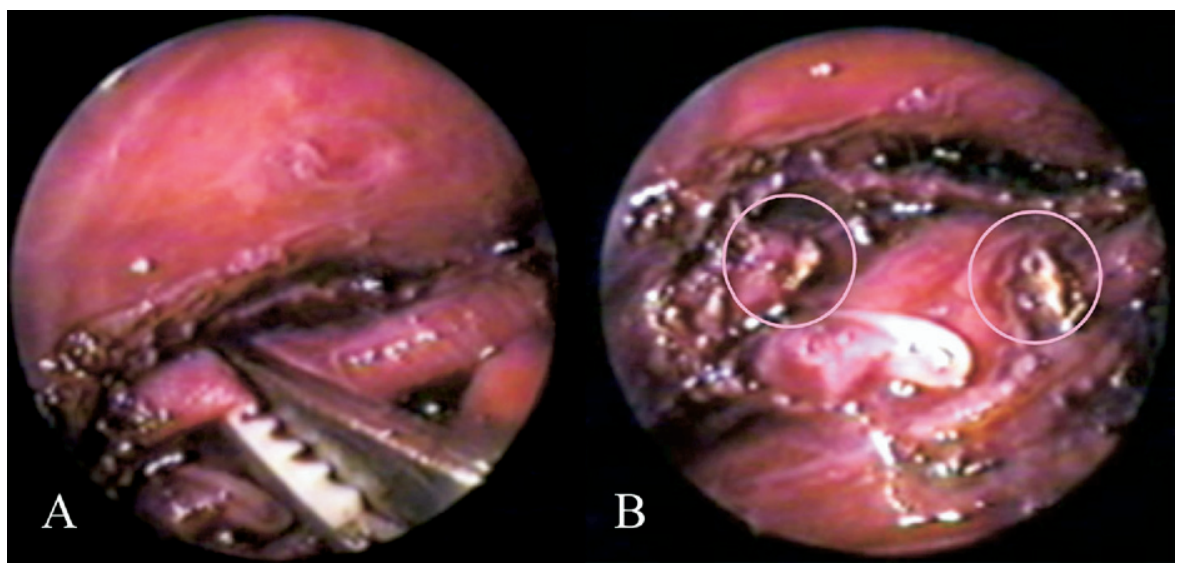

Figure 2. A, The 5-mm Harmonic scalpel is used to divide the posterior aortic arch, which has a 2- to $2.5-\mathrm{mm}$ diameter. $B$, The arch is divided, leaving two well-sealed stumps (circles). The posterior wall of the trachea is relieved from the compression, and the area for the esophageal anastomosis is now clear.

pending. On the basis of the currently available literature on the fields of vascular and general thoracic surgery, we think that thoracoscopy is safe if performed by experienced surgeons. Even when we are reporting a single case, we think that the Harmonic scalpel is a tool that can be safely used for the division of vascular rings of appropriate sizes.

\section{References}

1. Hartenberg MA, Salzberg AM, Krummel TM, Bush JJ. Double aortic arch associated with esophageal atresia and tracheoesophageal fistula. J Pediatr Surg. 1989;24:488-90.
2. Georghiou GP, Stamler A, Berman M, Sharoni E, Vidne BA, Sahar G. Advantages of the ultrasonic Harmonic Scalpel for radial artery harvesting. Asian Cardiovasc Thorac Ann. 2005;13:58-60.

3. Luciani N, Anselmi A, Gaudino M, Nasso G, Glieca F, Martinelli $\mathrm{L}$, et al. Harmonic scalpel reduces bleeding and postoperative complications in redo cardiac surgery. Ann Thorac Surg. 2005;80: 934-8.

4. Matsumoto K, Tsuneyoshi I, Iguro Y, Kinjo T, Yotsumoto G, Ueno M, et al. Effects of ultrasonic skeletonization on internal thoracic and gastroepiploic arteries for coronary artery bypass grafting. Eur J Cardiothorac Surg. 2006;30:592-6.

5. Laje P, Martinez-Ferro M, Grisoni E, Dudgeon D. Intra-abdominal pulmonary sequestration. A case series and review of the literature J Pediatr Surg. 2006;41:1309-12.

\section{Surgical management of giant left atrial diverticulum}

Jonathan McGuinness, AFRCSI, Ali Kindawi, FRCSI, Shaf Tajri, MRCS, Kevin Walsh, FRCPI, Lars Nolke, FRCSI, and Alfred E. Wood, FRCSI, Dublin, Ireland

\section{Clinical Summary}

After the incidental finding of a hyperdynamic precordium during a respiratory tract infection and subsequent note of cardiomegaly on a chest $\mathrm{x}$-ray film, an asymptomatic 11/2-year-old girl was referred for transthoracic echocardiography. This showed dex-

\footnotetext{
From the Department of Paediatric Cardiac Surgery and Cardiology, Our Lady's Hospital for Sick Children, Dublin, Ireland.

Received for publication May 2, 2006; accepted for publication Sept 6, 2006.

Address for reprints: Alfred E. Wood, FRCSI, Department of Paediatric Cardiac Surgery, Our Lady's Hospital for Sick Children, Crumlin, Dublin, Ireland (E-mail: jmcguinness2@ rcsi.ie).

J Thorac Cardiovasc Surg 2007;133:820-2

$0022-5223 / \$ 32.00$

Copyright $\odot 2007$ by The American Association for Thoracic Surgery doi:10.1016/j.jtcvs.2006.09.084
}

troposition of the heart secondary to a giant diverticulum of the left atrium (Figure 1,A). Owing to this compression and its risk of progression, combined with the risk of thromboembolism, surgical plication was considered.

Through a left posterolateral thoracotomy, the diverticulum was noted to extend over the left ventricle with the circumflex artery overlying it; therefore, only a selective atrioplasty was undertaken. Postoperatively, the diverticulum was still sizable, and magnetic resonance imaging demonstrated low-grade contraction within the wall of the diverticulum, communicating with the left atrium but not the left ventricle.

Eight months later, with the use of cardiopulmonary bypass, the diverticulum was excluded from the circulation through pericardial patch closure of its orifice from the left atrium. Ten hours postoperatively, circulatory arrest occurred, and the child was placed on extracorporeal membrane oxygenation (ECMO) support. An echocardiogram revealed poor biventricular function with clot filling the diverticulum. Cardiac catheterization revealed globally 